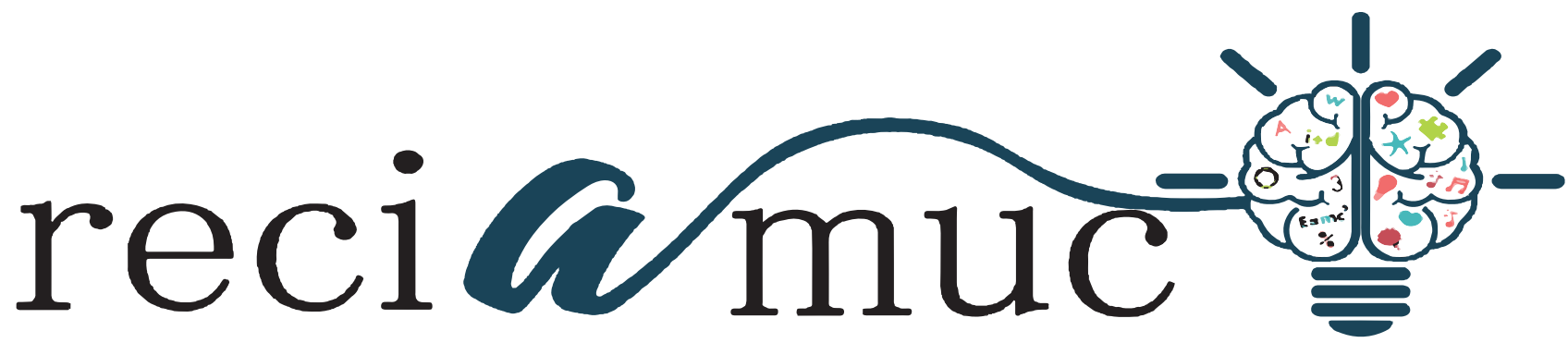

DOI: 10.26820/reciamuc/5.(4).noviembre.2021.307-313

URL: https://reciamuc.com/index.php/RECIAMUC/article/view/749

EDITORIAL: Saberes del Conocimiento

REVISTA: RECIAMUC

ISSN: 2588-0748

TIPO DE INVESTIGACIÓN: Reporte de caso

CÓDIGO UNESCO: 32 Ciencias Médicas

PAGINAS: $307-313$

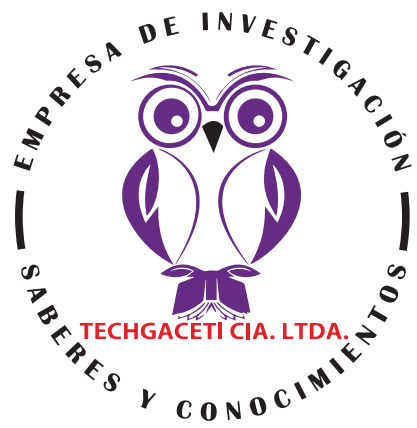

\title{
Aneurismas de arteria comunicante anterior. A propósito de caso clínico en Hospital clínica San Francisco
}

Anterior communicating artery aneurysms. Regarding a clinical case in San Francisco Clinical Hospital

Aneurismas da artéria comunicante anterior. Sobre um caso clínico no San Francisco Clinical Hospital

Eddy Xavier Díaz Recalde'; Mario Fernando De la Pared Ramírez;; Omar Josué Jaramillo Wong3; María Matilde Macías Sabando4

RECIBIDO: 15/09/2021 ACEPTADO: 05/10/2021 PUBLICADO: 29/11/2021

1. Médico; Investigador Independiente; Guayaquil, Ecuador; dr.eddydiazr@gmail.com; (DD https://orcid.org/00000002-7717-5437

2. Médico; Investigador Independiente; Guayaquil, Ecuador; drmario_11@hotmail.com; (D) https://orcid.org/00000002-1915-438X

3. Médico; Investigador Independiente; Guayaquil, Ecuador; dr.omar.jaramillo@gmail.com; (ID https://orcid.org/0000-0001-9204-602X

4. Médico; Investigador Independiente; Guayaquil, Ecuador; mati210390@gmail.com; (D) https://orcid.org/00000001-6398-6151

\section{CORRESPONDENCIA}

Eddy Xavier Díaz Recalde

dr.eddydiazr@gmail.com

Guayaquil, Ecuador

ㄷ RECIAMUC; Editorial Saberes del Conocimiento, 2021 


\section{RESUMEN}

Los aneurismas cerebrales son patologías frecuentes en la práctica neuroquirúrgicas, y teniendo un impacto socio económico por su morbimortalidad en el evento de ruptura. Los aneurismas de Comunicante anterior (AcomA) representan el 25-30\% de los aneurismas rotos, siendo los que mayor tasa de ruptura tienen dentro del circuito anterior. Se trata de paciente femenino de 51 años de. Refiere cefalea pulsátil de moderada intensidad hace 48 horas que se exacerbo agregándose nauseas que llevan al vomito en 2 ocasiones, se le realiza tomografía de cerebro evidenciando hemorragia subaracnoidea en cisternas de la base, cisuras silvianas de predominio izquierdo. Se realiza reconstrucción tomografica de cerebro en donde se observa aneurisma cerebral. Debido a ello y por el alto riesgo de ruptura de aneurisma cerebral y con ello coma, se decide ingreso a uci 3. En el caso de estudio se ha presentado una hemorragia subaracnoidea con clasificación de sangrado intracraneal Fisher II y una escala de severidad HUNT HESS grado I, como consecuencia de una aneurisma cerebral comunicante anterior, la sintomatología referida por la paciente al momento del ingreso al centro de salud es una cefalea moderada a intensa que se corresponde con lo descrito en la literatura, aunque la misma no presenta rigidez en la nuca. El procedimiento quirúrgico realizado fue embolizacion de aneurisma por medio de depósitos de espirales de platino (coils) colocados en el seno del aneurisma, tratamiento ampliamente utilizado y que va a acorde a lo descrito en la literatura. Sin embargo la paciente presenta otras aneurismas que requieren la colocación de stents, las cuales serán realizadas cuando la paciente este estable.

Palabras clave: Aneurisma, Roto, Embolización, Fisher, Cefalea.

\section{ABSTRACT}

Brain aneurysms are frequent pathologies in neurosurgical practice, and have a socio-economic impact due to their morbidity and mortality in the event of rupture. Anterior communicating aneurysms (AcomA) represent $25-30 \%$ of ruptured aneurysms, being the ones with the highest rupture rate within the anterior circuit. This is a 51-year-old female patient. She refers to a pulsatile headache of moderate intensity 48 hours ago that was exacerbated adding nausea that led to vomiting on 2 occasions; a brain tomography was performed showing subarachnoid hemorrhage in the basal cisterns, sylvian fissures predominantly on the left. Tomographic reconstruction of the brain is performed where a cerebral aneurysm is observed. Due to this and the high risk of cerebral aneurysm rupture and with it coma, admission to ICU 3 was decided. In the case study, a subarachnoid hemorrhage was presented with a Fisher II intracranial bleeding classification and a HUNT HESS severity scale. Grade I, as a consequence of an anterior communicating cerebral aneurysm, the symptomatology referred by the patient at the time of admission to the health center is a moderate to severe headache that corresponds to what is described in the literature, although it does not present rigidity in the nape. The surgical procedure performed was embolization of the aneurysm by means of platinum coil deposits (coils) placed within the aneurysm, which is a widely used treatment that is in accordance with that described in the literature. However, the patient has other aneurysms that require the placement of stents, which will be performed when the patient is stable.

Keywords: Aneurysm, Broken, Embolization, Fisher, Headache.

\section{RESUMO}

Os aneurismas cerebrais são patologias frequentes na prática neurocirúrgica e apresentam impacto socioeconômico devido à sua morbimortalidade em caso de ruptura. Os aneurismas comunicantes anteriores (AcomA) representam 25-30\% dos aneurismas rotos, sendo os que apresentam a maior taxa de rotura no circuito anterior. Esta é uma paciente de 51 anos. Refere-se a uma cefaleia pulsátil de intensidade moderada há 48 horas que foi exacerbada, acrescentando náusea que levou ao vômito em 2 ocasiões; Foi realizada tomografia de cérebro mostrando hemorragia subaracnóidea em cisternas basais, com fissuras silvianas predominantemente à esquerda. A reconstrução tomográfica do cérebro é realizada onde um aneurisma cerebral é observado. Por isso e pelo alto risco de ruptura do aneurisma cerebral e com ele coma, optou-se pela internação na UTI 3. No estudo de caso, uma hemorragia subaracnóidea foi apresentada com uma classificação de sangramento intracraniano de Fisher II e uma escala de gravidade HUNT HESS. Grau I, em consequência de um aneurisma cerebral comunicante anterior, a sintomatologia referida pelo paciente no momento da admissão no centro de saúde é uma cefaleia moderada a intensa que corresponde ao descrito na literatura, embora não apresente rigidez na nuca. O procedimento cirúrgico realizado foi a embolização do aneurisma por meio de depósitos (coils) de platina colocados no interior do aneurisma, tratamento amplamente utilizado e de acordo com o descrito na literatura. Porém, o paciente possui outros aneurismas que requerem a colocação de stents, que serão realizados quando o paciente estiver estável.

Palavras-chave: Aneurisma Quebrado, Embolização, Fisher, Dor de Cabeça. 


\section{ANEURISMAS DE ARTERIA COMUNICANTE ANTERIOR. A PROPÓSITO DE CASO CLÍNICO EN HOSPITAL CLÍNICA SAN FRANCISCO}

\section{Introducción}

Los aneurismas cerebrales son patologías frecuentes en la práctica neuroquirúrgicas, y teniendo un impacto socio económico por su morbimortalidad en el evento de ruptura. Los aneurismas de Comunicante anterior (AcomA) representan el 25-30\% de los aneurismas rotos, siendo los que mayor tasa de ruptura tienen dentro del circuito anterior (Olivero, Gavira, \& Cañas, 2020, pág. 140).

Estos últimos constituyen un reto especial para cualquier esfuerzo terapéutico, debido a la variabilidad anatómica que presentan y a las características hemodinámicas específicas que los caracterizan y llegan a ser considerados como los más complejos en la circulación anterior. Ellos constituyen un ejemplo muy particular de cómo la evolución en el conocimiento de la microanatomía neurovascular y el desarrollo y aplicación de nuevas tecnologías han influido en la toma de decisiones de los neurocirujanos (González González, Hernández Zayas, \& Verdial Vidal, 2006).

Cuando se produce una hemorragia subaracnoidea de causa aneurismática, esta acarrea la alta probabilidad de ocasionar complicaciones de distinta naturaleza, desde el momento mismo del ictus hasta pasados los 14 días, los que comprometen la vida del paciente, por los cambios que se generan en la dinámica del flujo sanguíneo cerebral, tanto por los cambios estructurales que ocasionan en la pared de las arterias, con cambios morfológicos por isquemia cerebral, o por afectación de la circulación del líquido cefalorraquídeo, que acaba en dilatación hidrocefálica aguda, resangrado, etc. que pueden ser motivo de muerte temprana (Coasaca-Torres, Loayza-Alarico, \& Navarrete-Mejia, 2018, pág. 30).

Las causas de este padecimiento incluyen trastornos degenerativos o adquiridos, poliquistosis, colagenopatías, ateroesclerosis, traumatismo, infección (micosis), malformaciones arteriovenosas (MAV), vasculitis y fármacos. Son lesiones típicas de los adul- tos, con pico de presentación entre los 40 y los 60 años. El riesgo de ruptura es de 1-2\% por año para aneurismas asintomáticos íntegros, existiendo una probabilidad mayor de ruptura en pacientes con aneurismas múltiples (Quezada Bucio, Arenas Ozuna, Gómez Gallardo, \& Molina Guerra, 2008, pág. 167).

La etiología de los aneurismas puede ser:

- Ateroesclerótica o hipertensiva: Es presuntamente la principal etiología de la mayoría de los aneurismas saculares, probablemente interactuando con la predisposición genética.

- Predisposición genética (por ejemplo, un defecto en la capa muscular de la pared arterial).

- Embolica: como en un mixoma auricular.

- Infecciosa (también llamados "aneurismas micóticos")

- Traumática.

- Asociada con otras condiciones.

Condiciones asociadas con los aneurismas

- Enfermedad de riñones poliquísticos autosómica dominante.

- Displasia fibromuscular (FMD): la prevalencia de aneurismas en la FMD renal es de $7 \%$, mientras que en FMD aortocraneal es de $21 \%$.

- Malformaciones arteriovenosas (AVM) incluyendo enfermedad de Moya-Moya.

- Alteraciones del tejido conectivo:

1. Ehlers-Danlos, especialmente el tipo IV (Deficiencia de colágeno tipo III) que también tiene una alta tasa de disección arterial incluso con angiografía o coiling.

2. Síndrome de Marfán.

3. Pseudoxantoma elástico. 
- Otros múltiples miembros de la familia con aneurismas intracraneales

- Síndrome familiar de aneurismas intracraneales (FIA): 2 o más familiares, de tercer grado o más cercanos, con aneurismas radiológicamente demostrados.

- Coartación de la aorta.

- Síndrome de Osler-Weber-Rendu.

(1) Ateroesclerosis.

- Endocarditis bacteriana (Rodas barrios, 2016, pág. 6).

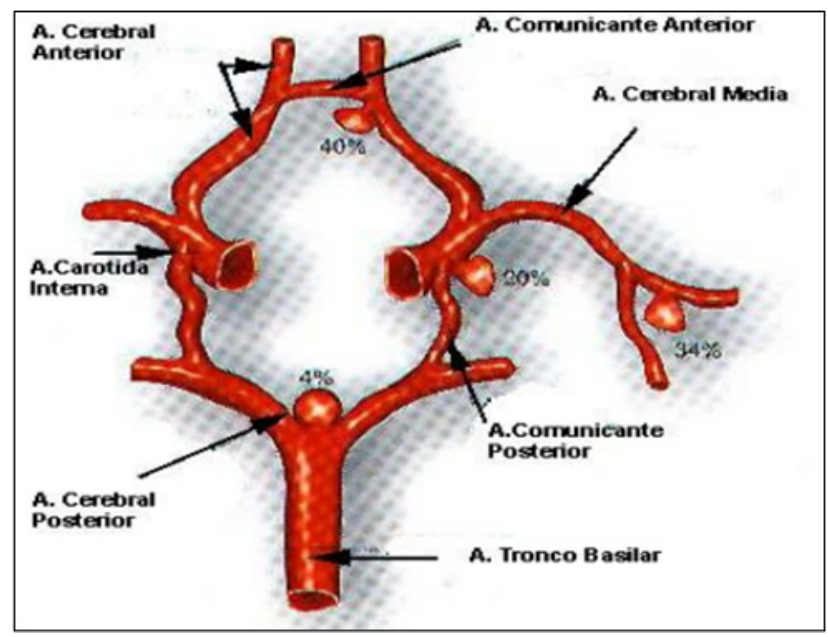

Imagen 1. Localización de las aneurismas

Fuente: (Rodas barrios, 2016)

\begin{tabular}{|c|c|}
\hline Grado & Descripción \\
\hline 0 & $\begin{array}{l}\text { Aneurisma no roto. } \\
\text { (Mortalidad Perioperatoria } 0-5 \% \text { ) }\end{array}$ \\
\hline 1 & $\begin{array}{l}\text { Asintomático o cefalea leve y rigidez nucal leve. } \\
\text { (Mortalidad Perioperatoria } 0-5 \% \text { ) }\end{array}$ \\
\hline $1 a$ & Sin reacción aguda meníngea o cerebral, pero con déficit neurológico \\
\hline 2 & $\begin{array}{l}\text { Parálisis de nervios craneales (III, IV), cefalea de moderada a severa y rigidez } \\
\text { nucal. } \\
\text { (Mortalidad Perioperatoria 2-10\%) }\end{array}$ \\
\hline 3 & $\begin{array}{l}\text { Déficit focal moderado, letargia o confusión (Mortalidad Perioperatoria 10- } \\
15 \% \text { ) }\end{array}$ \\
\hline 4 & $\begin{array}{l}\text { Estupor, hemiparesia severa o moderada, descerebración temprana. } \\
\text { (Mortalidad Perioperatoria } 60-70 \% \text { ) }\end{array}$ \\
\hline \multirow[t]{2}{*}{5} & $\begin{array}{l}\text { Coma profundo, rigidez de descerebración, apariencia moribunda. } \\
\text { (Mortalidad Perioperatoria } 70-100 \% \text { ) }\end{array}$ \\
\hline & $\begin{array}{l}\text { La presencia de enfermedad sistémica (HTA, DM, Aterosclerosis severa o } \\
\text { EPOC) así como evidencia de vasoespasmo severo en una arteriografia hará } \\
\text { que se aumente un punto el grado clínico del paciente }\end{array}$ \\
\hline
\end{tabular}

Imagen 2. Severidad de una hemorragia subaracnoidea no traumática según la clasificación de Hunt y Hess

Fuente: (Rodas barrios, 2016)

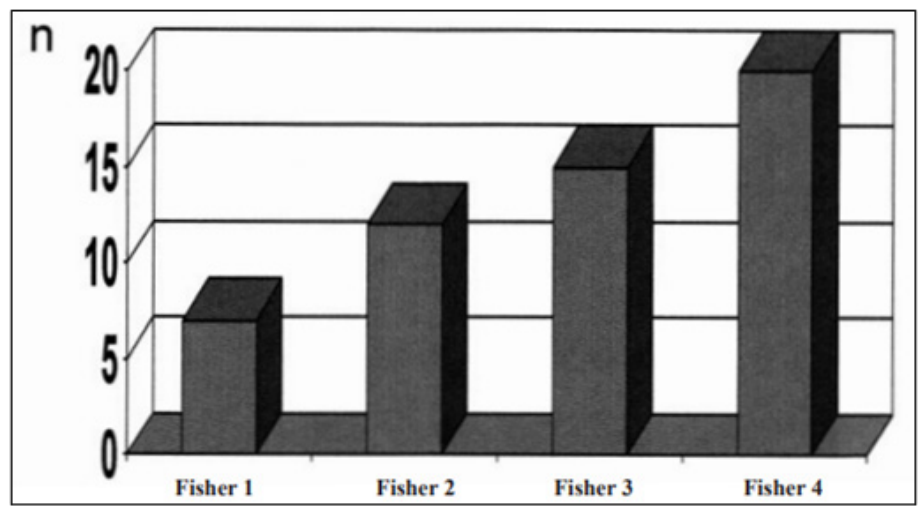

Imagen 3. Clasificación del sangrado intracraneal según la escala de Fisher.

Fuente: (González-Pérez, 2006) 


\section{ANEURISMAS DE ARTERIA COMUNICANTE ANTERIOR. A PROPÓSITO DE CASO CLÍNICO EN HOSPITAL CLÍNICA SAN FRANCISCO}

El éxito del tratamiento quirúrgico del aneurisma de AcomA es complicado por el hecho de que la arteria está situada en un área más profunda y rodeada por estructuras importantes tales como vasos sanguíneos mayores, dando por resultado un pronóstico pobre para los pacientes. En adición, la localización y la dirección del aneurisma depende de variables hemodinámicas tales como la dirección del flujo de la sangre y el tamaño del vaso sanguíneo. Todos estos factores son importantes al decidir el tratamiento quirúrgico y la conducta, agregando complejidad al procedimiento. El tratamiento quirúrgico y endovascular deben ser bien definidos en el manejo de estos aneurismas (Henrique Aguiar, y otros, s.f, pág. 8). El abordaje quirúrgico, es decir tomar la decisión de hacerlo, no debe considerar el tiempo transcurrido de la enfermedad, basta considerar algunas pautas seguras para operar, como la asociación del estado neurológico y el grado del Fisher, lo que se logra con el diagnóstico del grado de Hunt - Hess (Coasaca-Torres, Loayza-Alarico, \& Navarrete-Mejia, 2018, pág. 30).

\section{Caso clínico}

Se trata de paciente femenino de 51 años de edad con APP de obesidad e HTA. Refiere cefalea pulsátil de moderada intensidad hace 48 horas que se exacerbo agregándose nauseas que llevan al vomito en 2 ocasiones. Motivo por el cual es ingresada en otra casa de salud en donde realizan tomografía de cerebro evidenciando hemorragia subaracnoidea en cisternas de la base, cisuras silvianas de predominio izquierdo, por limitada capacidad resolutiva es transferida al Hospital clínica San francisco, para valoración y tratamiento integral especializado.

Al momento se recibe paciente, consiente, orientada en tiempo y espacio, aqueja cefalea intensa. Hemodinamicamente hipertensa con apoyo de drogas vasodilatadoras a base de nitroglicerina, con buena mecánica ventilatoria. Se realiza reconstrucción tomografica de cerebro en donde se observa aneurisma cerebral. Debido a ello y por el alto riesgo de ruptura de aneurisma cerebral y con ello coma, se decide ingreso a uci 3.

\section{Diagnóstico}

Hemorragia subaracnoidea FISHER II HUNT HESS I WFNS I.

\section{Examen físico}

\subsection{Neurológico}

Despierta orientada en tiempo y espacio con pupilas reactivas no focalidad motora sigue órdenes complejas Glasgow 15/15

\subsection{Cardiovascular}

Hemodinamicamente estable sin soporte vasopresor con RSCS rítmicos no soplos no tercer ruido no ruidos agregados con TAM 100 con FC 80 con buena perfusión periférica buen llenado capilar.

\subsection{Respiratoria}

Buena mecánica ventilatoria sin soporte de oxigeno con CSPS ventilados murmullo vesicular conservada no ruidos agregados

\subsection{Digestivo}

Abdomen blando depresible no doloroso con RHS presentes no catarsis no masas palpables no visceromeglaias no ruidos agregados.

\subsection{Renal / Metabólico}

Con diuresis conservada con azoados en urea 46 crea 0.6 con medio interno NA 144 K 3.9 CL 105

\subsection{Osteomuscular}

Extremidades simétricas no edema no ulcera de presión no escara.

\subsection{Infecciosos}

Afebril con marcadores infecciosos PCR 55 LEU 11 PROCAL 0.07 IL 8.2. 


\section{Medicación recibida}

- Ranitidina 50 mg IV cada 12 horas

- Paracetamol 1 gramo IV PRN

- Nimodipino 60 mg por vía oral cada 4 horas

- Losartan 100 mg VO cada día.

3. Otros exámenes

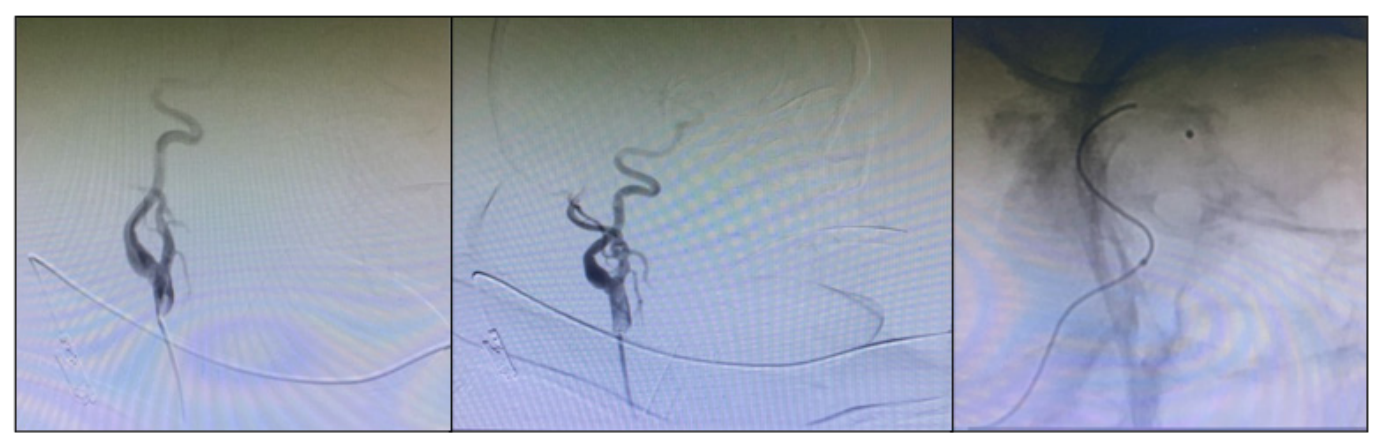

Imagen 4. Resonancia magnética de cráneo

Fuente: Los Autores

\subsection{Resonancia magnética de cráneo}

- Aneurisma de ACOA con estigmas de sangrado.

- Aneurisma de segmento cavernoso y oftálmico arteria carótida interna izquierda.

\section{Tratamiento}

\subsection{Procedimiento operatorio}

- Se realiza angioplastia con catéter punta balón y angioplastia química.

- Se procede a pasar sistemas a arteria carótida interna izquierda para estudio de aneurisma. Se observan 2 aneurismas en segmento cavernoso, uno fusiforme y la otra mala relación cuello domo.

- Aneurisma de segmento oftálmico con mala relación cuello domo, los cuales son indicativos de tratamiento con STENT diversor de flujo o STENT posterior a resolución de cuadro actual para indicar doble antiagreacion.

\subsection{Resumen de procedimiento quirúrgico}

- Embolizacion de aneurisma de complejo comunicante anterior.

\section{Discusión}

Paciente reportado de grave el cual se encuentra en cuidado hospitalario, el cual intercurre con hemorragia subaracnoidea la cual fue intervenida con colocación de coiIs y como hallazgo incidental 4 aneurismas más en espera de colocación de diversor, monitorización de su hemodinamia y función respiratoria se comunican novedades a familiares. En este contexto si se ha realizado el proceso de embolizacion y se requiere colocación de stents para el resto de las 4 aneurismas encontradas, lo que indica que la opción de otras técnicas menos agresivas están descartadas, lo que sugiere que estas aneurismas representan un grado de riesgo importante, sin embargo, como la condición del paciente sigue siendo grave, se desconoce el grado de severidad de las mismas, lo importante hasta ahora es que el paciente esta en constante monitoreo y 


\section{ANEURISMAS DE ARTERIA COMUNICANTE ANTERIOR. A PROPÓSITO DE CASO CLÍNICO EN HOSPITAL CLÍNICA SAN FRANCISCO}

no se ha presentado hasta el presente un desenlace fatal.

\section{Conclusión}

En el caso de estudio se ha presentado una hemorragia subaracnoidea con clasificación de sangrado intracraneal Fisher II y una escala de severidad HUNT HESS grado I, como consecuencia de una aneurisma cerebral, la sintomatología referida por la paciente al momento del ingreso al centro de salud es una cefalea moderada a intensa que se corresponde con lo descrito en la literatura, aunque la misma no presenta rigidez en la nuca. El procedimiento quirúrgico realizado fue embolizacion de aneurisma por medio de depósitos de espirales de platino (coils) colocados en el seno del aneurisma, tratamiento ampliamente utilizado y que va a acorde a lo descrito en la literatura. Sin embargo la paciente presenta otras aneurismas que requieren la colocación de stents, las cuales serán realizadas cuando la paciente este estable.

\section{Bibliografía}

Coasaca-Torres, J. A., Loayza-Alarico, M., \& Navarrete-Mejia, P. (2018). COMPLICACIONES POR ROTURA DE ANEURISMAS CEREBRALES EN PACIENTES OPERADOS EN UN HOSPITAL DE LIMA-PERÚ. 2006-2014. Revista de la Facultad de Medicina Humana, 18(1).

González González, J. L., Hernández Zayas, H., \& Verdial Vidal, R. (2006). Aneurismas del complejo arteria cerebral anterior / arteria. Rev Cubana Cir, 45(1).

González-Pérez, M. (2006). Resultado del tratamiento de la hemorragia subaracnoidea debida a rotura de aneurismas cerebrales. Neurocirugía, 17(5), 433-439.

Henrique Aguiar, P., Santana, P., França Bezerra, D., V. Silva, F., Panagopoulos, A., \& Vinícius Maldaun, M. (s.f). Aneurismas de la Arteria Comunicante Anterior: Manejo y Casuística.

Olivero, M., Gavira, M., \& Cañas, S. (2020). Aneurismas Comunicante Anterio. DEMO FLANC, 29(4), 138-148.

Quezada Bucio, H. G., Arenas Ozuna, J., Gómez GaIlardo, L., \& Molina Guerra, J. (2008). Aneurismas cerebrales. Localización mas frecuente en pacientes mexicanos estudiados en el Hospital de Especialidades del Centro Médico Nacional La Raza. Anales de Radiología de México, 3, 167-173.

Rodas barrios, R. E. (2016). Complicaciones en pacientes operados por aneurismas cerebrales.

\section{CITAR ESTE ARTICULO:}

Díaz Recalde, E. X., De la Pared Ramírez, M. F., Jaramillo Wong, O. J., \& Macías Sabando, M. M. (2021). Aneurismas de arteria comunicante anterior. A propósito de caso clínico en Hospital clínica San Francisco. RECIAMUC, 5(4), 307-313. https://doi.org/10.26820/reciamuc/5.(4).noviembre.2021.307-313

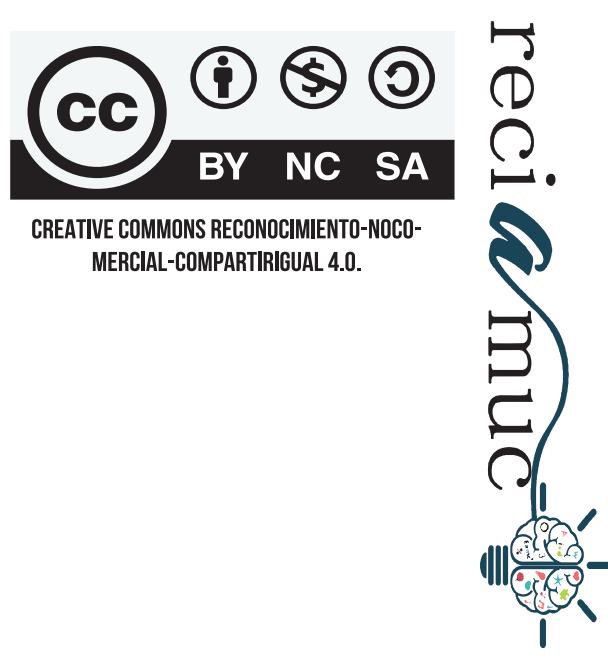

